(2) Open Access Full Text Article

ORIGINAL RESEARCH

\title{
Experimental Study on the Role of Apelin-13 in Alleviating Spinal Cord Ischemia Reperfusion Injury Through Suppressing Autophagy
}

This article was published in the following Dove Press journal:

Drug Design, Development and Therapy

\section{Zhewei Xu \\ Zhiyue Li}

Department of Orthopedics, Third Xiangya Hospital of Central South University, Changsha, Hunan 410013, People's Republic of China
Correspondence: Zhiyue Li Department of Orthopedics, Third Xiangya Hospital of Central South University, Changsha, Hunan 410013,

People's Republic of China

Tel +861378731 8116

Email oirieqnoh@sina.com
Background: This study aimed to explore the effect of Apelin-13 in protecting rats against spinal cord ischemia reperfusion injury (SCIR), as well as the related molecular mechanisms. Methods: One week prior to the experiment, experimental Sprague-Dawley rats were injected with Apelin-13 and the autophagy activator rapamycin through the tail vein once a day for 7 consecutive days. The SCIR rat model was prepared through the abdominal aorta clamping method. At $72 \mathrm{~h}$ after injury, the spinal cord tissue water content, infarct volume, and normal neuron count were determined to evaluate the degree of spinal cord tissue injury in the rats. The Basso-Beattie-Bresnahan scoring standard was adopted for functional scoring of the rat hind leg, to reflect the post-injury motor function. At $72 \mathrm{~h}$ after injury, changes in mitochondrial membrane potential, reactive oxygen species content, and mitochondrial ATP were detected. ELISA was carried out to detect the malonaldehyde content, as well as catalase, superoxide dismutase, and glutathione catalase activities in spinal cord tissues at $72 \mathrm{~h}$ after injury. Quantitative chemistry was conducted to examine the contents of nitric oxide (NO) and endothelial nitric oxide synthase (eNOS) in spinal cord tissues. Finally, the expression of autophagy-related proteins, Beclin1, ATG5, and LC3, in spinal cord tissues was detected through the Western blotting assay.

Results: Apelin-13 pretreatment alleviated SCIR, promoted motor function recovery, suppressed mitochondrial dysfunction, resisted oxidative stress, and inhibited autophagy in spinal cord tissues following ischemia reperfusion injury.

Conclusion: Apelin-3 exerts protection against SCIR by suppressing autophagy.

Keywords: Apelin-13, spinal cord ischemia reperfusion injury, autophagy, rapamycin, oxidative stress, mitochondrion

\section{Introduction}

Spinal cord ischemia reperfusion injury (SCIR) is a complication occurring under multiple pathophysiological states, which results in paralysis and paraplegia. ${ }^{1} \mathrm{SCIR}$ not only is a clinical issue, but also causes a huge social burden. Clinicians and scientific researchers have made great efforts to develop various measures, such as extracorporeal shunt or bypass techniques, ${ }^{2}$ cerebrospinal fluid (CSF) drainage, ${ }^{3}$ retrograde venous perfusion, ${ }^{4}$ intercostal arterial reconstruction, and drug therapy (eg, steroids, free radical scavengers and vasodilators), to alleviate and treat SCIRinduced damage. However, these methods cannot achieve satisfactory therapeutic effects; therefore, there is an urgent need to develop a new effective treatment for SCIR. 
Autophagy exerts a vital role in cell survival at the time of metabolic stress, and the maintenance of metabolism of cytoplasmic components through the autolysosome pathway. ${ }^{5,6}$ Some studies have explored the role of autophagy in the SCIR process, but the results are mostly inconsistent and even contradictory. For example, one study reports that, after ischemia reperfusion injury, the activation and enhancement of autophagy induce nerve injury, ${ }^{7}$ whereas another reports that autophagy promotes neuron death after ischemia reperfusion injury, and that suppressing autophagy exerts the protective effect. ${ }^{8}$ All in all, the precise mechanism of action of autophagy in SCIR remains to be further investigated.

Apelin-13 is a newly discovered polypeptide, which exerts an important neuroprotective effect in the central nervous system (CNS). ${ }^{9}$ Neuroprotection by Apelin-13 in ischemia reperfusion treatment has been verified. In ischemia reperfusion and traumatic brain injury (TBI), Apelin-13 shows a certain protective effect; in particular, it suppresses autophagy to reduce TBI-induced neuron death. ${ }^{10}$ However, no existing studies have reported that Apelin-13 alleviates SCIR nerve injury through regulating the autophagy pathway.

This study constructed an SCIR rat model after Apelin13 pretreatment, analyzed the protective effect of Apelin13 pretreatment on spinal cord nerves after SCIR, as well asvariations in autophagy, and explored the mechanism of action of autophagy in SCIR, to provide new therapeutic targets for, and new ways of thinking about, SCIR.

\section{Materials and Methods}

\section{Materials}

Apelin-13 was purchased from Sigma Aldrich (St Louis, MO). Malonaldehyde (MDA), catalase (CAT), superoxide dismutase (SOD), glutathione (GSH), and enzyme-linked immunosorbent assay (ELISA) kits were bought from Abcam Inc. (Cambridge, MA). Goat anti-rat endothelial nitric oxide synthase (eNOS), Bax primary antibody, mouse anti-rat Bcl-2 primary antibody (Santa Cruz), mouse anti-goat secondary antibody, and rabbit anti-mouse secondary antibody were provided by Santa Cruz Biotechnology (Dallas, TX).

\section{Experimental Animals}

All of the procedures were carried out in accordance with the Chinese Guidelines for Animal Welfare and Experimental Protocol and were approved by the Animal Care and Use Committee of the Third Xiangya Hospital, Central South
University. In brief, 35 healthy Sprague-Dawley (SD) male rats (10-12 weeks old, weighing $250 \pm 20 \mathrm{~g}$ ) were purchased from Silaikejingda Company (Hunan Province, People's Republic of China), and raised in constant temperature (at about $25^{\circ} \mathrm{C}$ ) animal pens under a $12 \mathrm{~h}-12$ hlight-dark cycle. All animals had free access to standard granulated feed and water. Then, all animals were randomized into four groups: sham operation group, ischemia reperfusion group, Apelin-13 pretreatment group, and RAPA+Apelin-13 pretreatment group, with eight in each group, while the remaining three rats were used for standby application.

\section{Methods}

\section{Experimental Grouping}

Group A (sham operation group, $\mathrm{n}=8$ ): only the abdominal aorta was exposed, while the blood flow was not blocked;

Group B (ischemia reperfusion group, $\mathrm{n}=8$ ): injection with normal saline (NS) through the tail vein $10 \mathrm{~min}$ prior to perfusion;

Group C (Apelin-13 pretreatment group, $n=8$ ): injection with Apelin-13 through the tail vein 1 week prior to perfusion, once a day for 7 consecutive days.

Group D (RAPA+Apelin-13 pretreatment group, $\mathrm{n}=8$ ): injection with $0.1 \mathrm{mg} / \mathrm{kg}$ Apelin-13 and $2 \mathrm{mg} / \mathrm{kg}$ autophagy activator rapamycin through the tail vein 1 week prior to perfusion, once a day for 7 consecutive days.

\section{Construction of the Experimental SCIR Model}

Each healthy male SD rat was given an intraperitoneal injection with $6 \%$ chloral hydrate $(6 \mathrm{~mL} / \mathrm{kg})$ for anesthesia; afterwards, each rat was given an intraperitoneal injection with morphine $(5 \mathrm{mg} / \mathrm{kg})$ for analgesia. Then, each animal was placed on the operating table in the supine position, and a small animal monitor and heating plate were connected to maintain the body temperature. An incision was made in the center of the abdomen, then the abdominal aorta was exposed and clamped using a non-invasive bulldog clamp at about $1 \mathrm{~cm}$ below the left renal artery and below the right renal artery branch. The disappearance of abdominal aorta pulsation below the clamp was confirmed, and the artery clamp was released after $25 \mathrm{~min}$ to initiate perfusion. The palpation of vascular pulsation in the clamped site suggested successful reperfusion; then, the wound was closed layer by layer, and erythromycin ointment was applied outside the wound. During the model preparation process, arterial blood was collected from the animal 10 min before clamping and $10 \mathrm{~min}$ after reperfusion, 
to detect blood glucose, oxygen partial pressure $\left(\mathrm{PaO}_{2}\right)$, partial pressure of carbon dioxide $\left(\mathrm{PaCO}_{2}\right)$, and $\mathrm{pH}$.

\section{Determination of Spinal Cord Water Content}

The water content of spinal cord tissue was determined by the dry/wet weight method. The spinal cord tissue immediately after animal sacrifice was measured as the wet weight, while the weight after drying for $48 \mathrm{~h}$ at $100^{\circ} \mathrm{C}$ was denoted as the dry weight. The water content was calculated according to the formula: $(1-$ dry weight/wet weight $) * 100 \%$.

\section{Determination of Spinal Cord Infarct Volume}

The spinal cord infarct volume was determined using the 2,3,5-triphenyltetrazolium chloride (TTC) staining method. In brief, the fresh spinal cord tissues were collected from rats immediately after they were killed, then frozen and sectioned into $1 \mathrm{~mm}$ slices, followed by TTC staining for $20 \mathrm{~min}$ at $37^{\circ}$ $\mathrm{C}$ and $4 \%$ paraformaldehyde fixation overnight. The infarct volume was analyzed, calculated using Adobe Systems analysis software, and expressed as a percentage.

\section{HE Staining}

The paraffin-embedded lumbar L2-L4 spinal cord tissues were fixed in $4 \%$ paraformaldehyde for $48 \mathrm{~h}$, sliced serially into $5 \mu \mathrm{m}$ sections, and attached onto glass slides pretreated with polylysine anti-slice escaping agent. The sections were then subjected to routine deparaffinization, hydration, and hematoxylin and eosin (HE) staining, in succession. Morphological changes in the spinal cord tissue were observed under an optical microscope, and the normal neurons were counted. According to the method proposed by Liu et al, ${ }^{11}$ the neurons were distinguished from glial cells and inflammatory cells based on neuron size, shape, and Nissl body aggregation. Cells with multipolar cell bodies, granular cytoplasm, and cell nuclei containing the nucleoli belonged to neurons, regardless of the cell size, while those with the typical cell nuclei of astrocytes, oligodendrocytes, and microglial cells were excluded (Figure 1). Three sections were selected for each rat, and 10 high-power fields of view were selected from each section to count the number of neurons.

\section{Analysis on the Motor Functions of Model Rats}

The motor functional behaviors after recovery from spinal cord injury were analyzed using the Basso-Beattie-Bresnahan

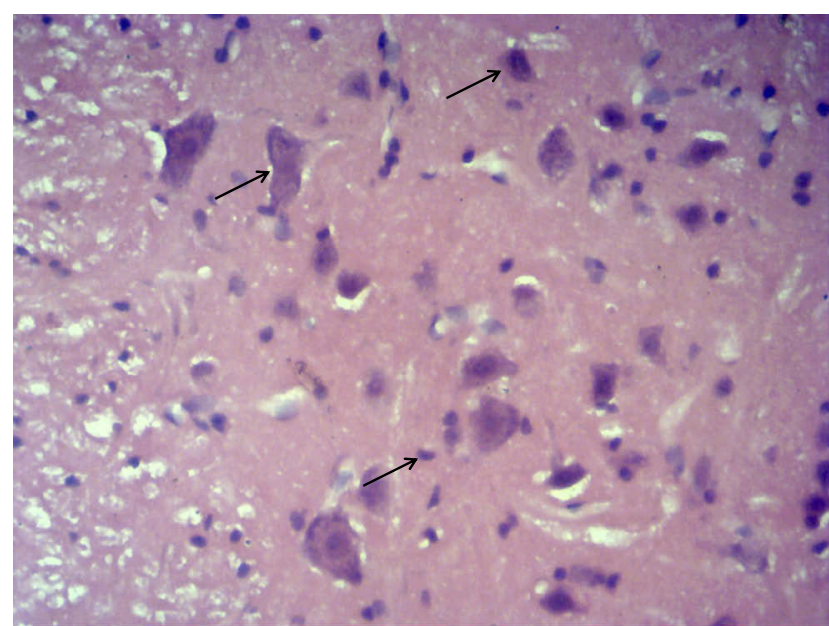

Figure I Representative picture of neurons by HE staining. Note: The black arrow points to the normal neurons.

(BBB) Scale formulated by Basso et $\mathrm{al}^{12}$. This scale is used to observe the motion and gait of rat limbs in an open site and the entire observation lasts for $4 \mathrm{~min}$. On this scale, 0 indicates the minimum score, while 21 represents the highest score. In this study, the BBB Scale scores before injury, and 1 and 3 days after surgery, were evaluated by two reviewers blind to the experiment.

\section{Specimen Collection}

On day 3 post-surgery, after the motor functions had been assessed, the rats were anesthetized again with chloral hydrate, and then the thoracic cavity was cut open at the sternum to expose the heart. A perfusion needle was inserted into the rat aortic root, then $1000 \mathrm{~mL}$ NS was used for perfusion and $500 \mathrm{~mL}$ of $10 \%$ formalin solution was used to fix tissues. After perfusion and fixation, the spinal cord was exposed on the back side, and the spinal cord tissues at the lumbar enlargement (L2-L4) site were extracted and divided into two segments: one was fixed in $4 \%$ paraformaldehyde and the other was preserved in liquid nitrogen.

\section{Detection of MDA Content, As Well As CAT, SOD, GSH-px, NO, and eNOS Activities in Spinal Cord Tissue of Each Group}

On day 3 after modeling, the spinal cord tissues at lumbar segment L2-L4 were dissected, sufficiently ground, digested, and centrifuged at $1000 \mathrm{rpm}$ for $10 \mathrm{~min}$ to collect the supernatants. Then, the malonaldehyde (MDA) content, and the 
activities of catalase (CAT), superoxide dismutase (SOD), glutathione catalase (GSH-px), nitric oxide (NO), and endothelial nitric oxide synthase (eNOS), were determined in accordance with the ELISA kit instructions and quantitative chemistry using the microplate reader.

\section{Detection of Mitochondrial Function}

The animals were killed on day 3 after modeling, the spinal cord tissues were dissected, and the mitochondrial component was isolated from the spinal cord tissue by density differential centrifugation. Mitochondrial membrane potential (MMP was determined through 5,5',6,6'tetrachloro-1,1',3,3'-tetraethylbenzimidazolcarbocyanine iodide (JC-1) staining, the production of mitochondrial reactive oxygen species (ROS) was detected through 2'7'dichlorofluorescein diacetate (DCF) staining, while the mitochondrial ATP content was measured through reversephase high-pressure liquid chromatography.

\section{Expression of Autophagy-Related Proteins in Spinal Cord Tissues Detected by Western Blotting}

Proteins were extracted from the spinal cord $\left(4^{\circ} \mathrm{C}\right)$ using protein extraction reagent and the protein concentration was quantified using a BCA protein assay kit according to the manufacturer's protocols. The Western blotting samples were prepared and subjected to sodium dodecyl sulfate-polyacrylamide gel electrophoresis (SDS-PAGE). After terminating electrophoresis, the samples were transferred onto polyvinylidene fluoride (PVDF) membranes at a constant pressure of $100 \mathrm{~V}$ for $1.5 \mathrm{~h}$. Subsequently, the PVDF membranes were blocked with 5\% skim milk in TBST for $2 \mathrm{~h}$, then corresponding antibodies (Beclin1, Atg5, and LC3) were added, and membranes were incubated at $4{ }^{\circ} \mathrm{C}$ overnight. On the following day, the secondary antibody was added after membrane washing to incubate for $1.5 \mathrm{~h}$; afterwards, the membrane was washed for $10 \mathrm{~min}$ four times and exposed to the gel imaging system. The experiment was repeated three times.

\section{Statistical Methods}

SPSS 20.0 software was adopted for statistical analyses. All continuous variables were expressed as mean \pm standard deviation. One-way analysis of variance (ANOVA) was used to compare the differences in continuous variables among the four groups. A $P$-value of $<0.05$ indicated statistical significance.

\section{Results}

\section{Apelin- 3 Had No Effect on Physiological} Indices in Rats Before and After Ischemia

During the spinal cord ischemia process, the differences in $\mathrm{PaO}_{2}, \mathrm{PaCO}_{2}, \mathrm{pH}$, and anal temperature in each group were not statistically significant, and the anal temperature during surgery was maintained at $38-39^{\circ} \mathrm{C}$. These results suggested that Apelin-13 and the autophagy activator rapamycin (RAPA), at the concentrations used in this experiment, had no influence on the blood glucose or blood gas of the rats $(P>0.05$, no statistically significant difference $)$ (Table 1).

\section{Apelin-I 3 Alleviated Nerve Injury After SCIR}

Spinal tissue water content, infarct volume, and normal neuron count are the main indices used to assess the degree of spinal cord tissue injury. On day 3 after surgery, the spinal cord tissue water contents in the sham operation group, model group, Apelin-13 pretreatment group, and RAPA+Apelin-13 pretreatment group were $70.1 \pm 3.4 \%, \quad 87.3 \pm 1.7 \%, \quad 74.3 \pm 1.2 \%, \quad$ and $\quad 82.4 \pm 2.8 \%$, respectively. No infarct lesions were seen in the spinal cord specimens of the sham operation group, whereas the spinal cord infarct volumes in the model group, Apelin13 pretreatment group, and RAPA+Apelin-13 pretreatment group were $46.8 \pm 2.6 \%, 21.3 \pm 3.97 \%$, and 40.2 $\pm 3.1 \%$, respectively. Meanwhile, the numbers of normal neurons in the sham operation group, model group, Apelin-13 pretreatment group, and RAPA+Apelin-13 pretreatment group were $40.1 \pm 2.8,11.7 \pm 2.4,36.5 \pm 3.1$, and $19.3 \pm 2.4$, respectively.

The spinal cord water content in the model group was higher than that in the sham operation group, and the normal neuron count was lower than that in the sham operation group $(P<0.05)$, revealing the success in modeling. Meanwhile, the spinal cord water content and infarct volume in the Apelin-13 pretreatment group were lower than those in the model group $(P<0.05)$, whereas the normal neuron count was higher than that in the model group $(P<0.05)$, suggesting that Apelin-13 protected against the spinal cord injury induced by ischemia reperfusion. At the same time, the neuron number in the RAPA+Apelin-13 pretreatment group was higher than that in the Apelin-13 pretreatment group $(P<0.05)$ (Figure $2 \mathrm{~A}-\mathrm{C})$, which demonstrated that the autophagy activator reversed the protective 
Table I Comparisons of Physiological Indices in SD Rats Before and After Ischemia $(\bar{x} \pm s n=8)$

\begin{tabular}{|l|l|l|l|l|}
\hline Group & Group A & Group B & Group C & Group D \\
\hline At I0 min Before Arterial Occlusion & & & & \\
$\quad \mathrm{Blood}$ glucose $(\mathrm{mmol} / \mathrm{L})$ & $3.84 \pm 1.06$ & $4.12 \pm 0.79$ & $3.88 \pm 0.64$ & $4.02 \pm 0.89$ \\
$\mathrm{PaO}_{2}(\mathrm{mmHg})$ & $106.29 \pm 7.12$ & $108.44 \pm 9.72$ & $109.27 \pm 7.21$ & $108.23 \pm 5.89$ \\
$\mathrm{PaCO}_{2}(\mathrm{mmHg})$ & $36.33 \pm 2.59$ & $40.14 \pm 4.72$ & $38.35 \pm 4.68$ & $39.72 \pm 1.64$ \\
$\mathrm{pH}$ & $7.35 \pm 0.06$ & $7.22 \pm 0.06$ & $7.44 \pm 0.08$ & $7.39 \pm 0.07$ \\
Anal temperature $\left({ }^{\circ} \mathrm{C}\right)$ & $38.71 \pm 0.14$ & $38.69 \pm 0.28$ & $38.57 \pm 0.17$ & $38.62 \pm 0.31$ \\
\hline Occlusion Period & & & & \\
$\mathrm{Blood}$ glucose $(\mathrm{mmol} / \mathrm{L})$ & $3.57 \pm 1.27$ & $3.67 \pm 0.84$ & $3.94 \pm 0.68$ & $3.88 \pm 0.72$ \\
$\mathrm{PaO}{ }_{2}(\mathrm{mmHg})$ & $101.27 \pm 10.96$ & $102.07 \pm 8.64$ & $107.24 \pm 7.86$ & $101.34 \pm 6.93$ \\
$\mathrm{PaCO} 2(\mathrm{mmHg})$ & $33.04 \pm 2.84$ & $34.87 \pm 2.67$ & $34.20 \pm 1.67$ & $34.02 \pm 2.11$ \\
$\mathrm{PH}$ & $7.34 \pm 0.06$ & $7.35 \pm 0.05$ & $7.39 \pm 0.04$ & $7.37 \pm 0.06$ \\
$\mathrm{Anal}$ temperature $\left({ }^{\circ} \mathrm{C}\right)$ & $38.37 \pm 0.27$ & $38.38 \pm 0.19$ & $38.40 \pm 0.09$ & $38.69 \pm 0.42$ \\
\hline 10 min of Reperfusion & & & & \\
$\mathrm{Blood}$ glucose $(\mathrm{mmol} / \mathrm{L})$ & $3.67 \pm 0.67$ & $3.69 \pm 0.77$ & $4.18 \pm 1.27$ & $3.94 \pm 0.86$ \\
$\mathrm{PaO}$ (mmHg) & $105.13 \pm 4.55$ & $106.23 \pm 7.93$ & $100.05 \pm 4.93$ & $104.98 \pm 3.72$ \\
$\mathrm{PaCO}$ (mmHg) & $34.21 \pm 1.67$ & $34.31 \pm 2.67$ & $34.05 \pm 2.54$ & $34.18 \pm 3.28$ \\
$\mathrm{pH}$ & $7.36 \pm 0.03$ & $7.37 \pm 0.05$ & $7.35 \pm 0.06$ & $7.38 \pm 0.09$ \\
$\mathrm{Anal}$ temperature $\left({ }^{\circ} \mathrm{C}\right)$ & $38.37 \pm 0.18$ & $37.48 \pm 0.16$ & $38.65 \pm 0.15$ & $38.12 \pm 0.47$ \\
\hline
\end{tabular}

effect of Apelin-13, revealing that Apelin-13 may exert spinal cord protection by suppressing autophagy.

\section{Apelin-I 3 Promoted Motor Function Recovery in Rats After SCIR}

In this study, the BBB Scale was applied to assess the hind leg function scores in the four groups of rats on days 1 and 3 after surgery, to evaluate the motor function recovery in rats after SCIR. A score of 21 points indicated the normal status of the hind leg, while 0 points suggested paralysis. On day 1 after surgery, the lower limb motion function score in the model group was apparently lower than that in the sham operation group $(t=23.8, P<0.05)$, that in Apelin-
13 pretreatment group was higher than that in the model group ( $t=3.9, P<0.05$ ), and that in the RAPA+Apelin-13 pretreatment group was lower than that in the Apelin-13 pretreatment group $(t=4.2, P<0.05)$. On day 3 after surgery, the lower limb motor function scores in the four groups of rats were higher than those on day $1(P<0.05)$; the score in the model group was lower than that in the sham operation group $(P<0.05)$, that in the Apelin-13 pretreatment group was higher than that in the model group ( $t=4.9, P<0.05)$, and the difference was not significant compared with the sham operation group $(P>0.05)$. Meanwhile, that in the RAPA+Apelin-13 pretreatment group was lower than that in the Apelin-13 pretreatment group $(t=5.1, P<0.05)$ (Table 2, Figure 3).
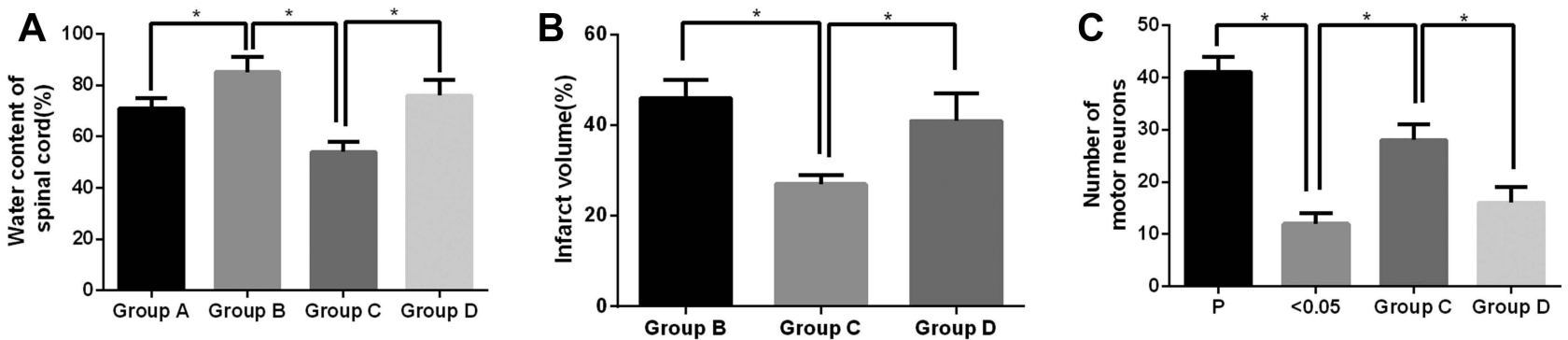

Figure 2 Effects of Apelin-13 and RAPA pretreatment alone or in combination on the spinal cord water content (A), infarct volume (B), and normal neuron count (C) in SCIR rats $(n=8)$. The asterisk $(*)$ indicates a significant difference $(P<0.05)$ between the two groups. 
Table 2 BBB Score (Points) in Each Group $(\bar{x} \pm s n=8)$

\begin{tabular}{|l|l|l|}
\hline Group & $\begin{array}{l}\text { On Day I After } \\
\text { Surgery }\end{array}$ & $\begin{array}{l}\text { On Day 3 After } \\
\text { Surgery }\end{array}$ \\
\hline Sham operation group & $17.2 \pm 2.4$ & $20.1 \pm 1.8$ \\
Model group & $4.2 \pm 1.6^{*}$ & $7.8 \pm 1.3^{*}$ \\
Apelin-13 pretreatment & $12.4 \pm 1.9^{\#}$ & $19.8 \pm 1.2^{\#}$ \\
group & & \\
RAPA+Apelin-13 & $6.4 \pm 2.1^{\&}$ & $9.3 \pm 1.8^{\&}$ \\
Pretreatment group & & 17.3 \\
F & 12.9 & 0.001 \\
$P$ & 0.013 & \\
\hline
\end{tabular}

Notes: Compared with sham operation group, $* P<0.05$; compared with model group, ${ }^{\#} P<0.05$; compared with Apelin-13 pretreatment group, ${ }^{8} P<0.05$.

\section{Apelin- 3 Protected Rats from SCIR By Resisting the Oxidative Stress (OS) Response}

On day 3 after surgery, the MDA, NO, and eNOS contents in spinal cord tissues of the model group were remarkably higher than those in the sham operation group $(t=9.8, t=12.8, t=11.7$, $P<0.05)$; those in the Apelin-13 pretreatment group were notably lower than those in the model group $(t=10.8, t=9.5$, $t=13.6, P<0.05$ ), and those in the RAPA+Apelin-13 pretreatment group were lower than those in the Apelin-13 pretreatment group $(t=9.2, t=7.4, t=8.1, P<0.05)$. The CAT, SOD, and GSH-px activities in spinal cord tissues of the model group were evidently lower than those in the sham operation group $(t=14.7, t=10.8, t=12.6, P<0.05)$; those in the Apelin-13 pretreatment group were dramatically higher than those in the model group ( $t=9.4, t=12.6, t=10.8, P<0.05$ ), and those in the RAPA+Apelin-13 pretreatment group were lower than

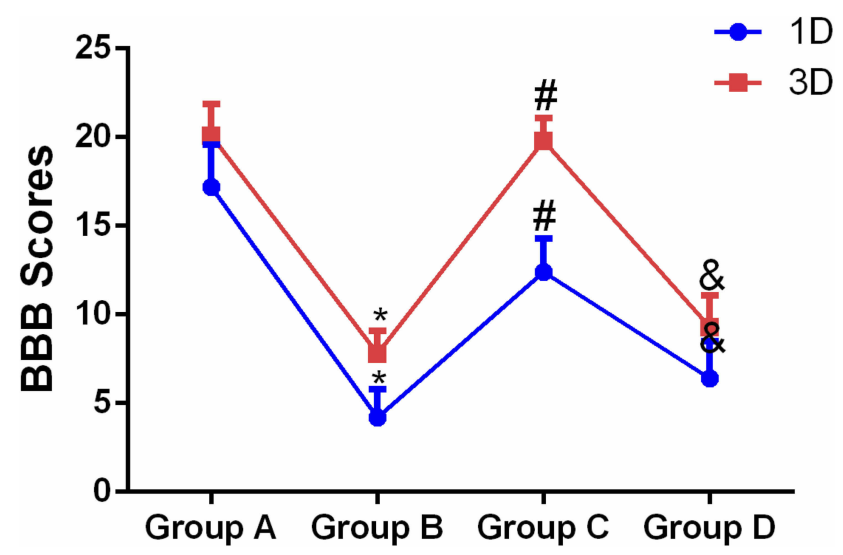

Figure 3 Comparisons of hind leg motor functions in four groups of rats on days I and 3 after SCIR $(n=8)$.

Notes: Compared with sham operation group, $* P<0.05$; compared with model group, ${ }^{\#} P<0.05$; compared with Apelin- 13 pretreatment group, ${ }^{\&} P<0.05$. those in the Apelin-13 pretreatment group ( $t=10.2, t=8.5$, $t=9.4, P<0.05$ ) (Table 3, Figure 4).

\section{Apelin-I 3 Suppressed Mitochondrial Injury Resulting from SCIR}

Rats in each group were killed 3 days after SCIR, then the MMP, ROS contents, and mitochondrial ATP levels were compared to analyze the effects of Apelin-13 on mitochondrial injury induced by SCIR. Compared with the sham operation group, MMP in the model group was markedly reduced, ROS production increased, while ATP production was reduced $(P<0.05)$, revealing that the mitochondrial function in spinal cord tissues of the model group was severely impaired. The MMP in the Apelin-13 pretreatment group evidently increased, ROS production was reduced, and the mitochondrial ATP level was maintained $(P<0.05)$, suggesting that Apelin-13 suppressed the SCIRinduced mitochondrial injury, while the protective effect of Apelin-13 on mitochondria was reversed in the RAPA +Apelin-13 pretreatment group (Figure 5A-C).

\section{Apelin- I 3 Suppressed Autophagy to Protect Rats from SCIR}

On day 3 after modeling, proteins were extracted from the spinal cord tissues of the four groups, and the expression of the autophagy-related proteins Beclin1, Atg5, and LC3 was detected through Western blotting. The results suggested that the expression of the three autophagy-related proteins Beclin1, Atg5 and LC3-II in model group B was markedly up-regulated, revealing that the autophagy levels in rats spinal cord tissues were abnormally elevated after SCIR. Meanwhile, the expression of Beclin1, Atg5, and LC3-II in the Apelin-13 pretreatment group was lower than that in the model group, indicating that Apelin-13 pretreatment alleviated the abnormally elevated autophagy induced by SCIR. In addition, when the expression of LC3-II in the RAPA+Apelin-13 pretreatment group was up-regulated, the expression of these three proteins was markedly down-regulated, revealing that autophagy activation reversed the protective effect of Apelin-13 (Figure 6A and B).

\section{Discussion}

In clinical practice, patients who have to undergo thoracic and abdominal aortic surgery as a result of disease are faced with the major complication of nerve injury induced by spinal cord ischemia. With the astounding advances in 
Table 3 Expression Levels of OS Factors in Spinal Cord Tissues of four Groups of Rats $(\bar{x} \pm s n=8)$

\begin{tabular}{|c|c|c|c|c|c|c|}
\hline Group & MDA (nmol/L) & CAT (U/mg) & SOD (U/mg) & GSH-px (U/mg) & $\mathrm{NO}(\mathrm{mmol} / \mathrm{L})$ & eNOS (ng/L) \\
\hline Sham operation group & $3.4 \pm 0.8$ & $9.9 \pm 1.7$ & $16.8 \pm 1.4$ & $28.6 \pm 3.1$ & $42.7 \pm 6.5$ & $16.8 \pm 1.5$ \\
\hline Model group & $12.8 \pm 1.4^{*}$ & $3.4 \pm 0.6^{*}$ & $6.5 \pm 0.8 *$ & $11.4 \pm 1.5^{*}$ & $139.1 \pm 9.2^{*}$ & $68.9 \pm 3.6 *$ \\
\hline Apelin-13 pretreatment group & $5.9 \pm 0.7^{\#}$ & $7.3 \pm 1.4^{\#}$ & $12.3 \pm 1.2^{\#}$ & $21.3 \pm 2.1^{\#}$ & $78.5 \pm 11.3^{\#}$ & $24.5 \pm 2.7^{\#}$ \\
\hline RAPA+Apelin-13 pretreatment group & $11.4 \pm 2.4^{\&}$ & $5.6 \pm 0.7^{\&}$ & $8.1 \pm 1.1^{\&}$ & $15.2 \pm 2.6^{8}$ & $106.3 \pm 19.8^{\&}$ & $47.8 \pm 3.5^{\&}$ \\
\hline$F$ & 12.7 & 9. 8 & 21.1 & 10.5 & 15.1 & 20.6 \\
\hline$P$ & 0.018 & 0.024 & 0.005 & 0.009 & 0.006 & 0.001 \\
\hline
\end{tabular}

Notes: Compared with sham operation group, ${ }^{*} P<0.05$; compared with model group, ${ }^{\#} P<0.05$; compared with Apelin- 13 pretreatment group, ${ }^{\circledR} P<0.05$.

physiology, pathology, and molecular biology over the past 10 years, the causes and processes of spinal cord injury are being gradually understood. Besides, numerous countermeasures and drugs have been developed in clinical application. This has substantially reduced the incidence rates of complications such as lower limb paralysis and hemiplegia, although they still occur sometimes. To further understand the pathophysiological changes in spinal cord ischemia, more reliable and repeatable experiments and clinical monitoring techniques are required. In this experiment, the SD rat model was selected to simulate the intraoperative aortic occlusion process and the resultant spinal cord injury. Using this experimental animal model, rats underwent transient aortic occlusion for $25 \mathrm{~min}$ to induce the first $30 \mathrm{~min}$ of SCIR, and the tail-vein injection of Apelin-13 protected the spinal cord. The experimental results suggested that Apelin-13 exerted spinal cord protection, which not only reduced the nerve injury after SCIR, but also promoted the recovery of motor function in rats after SCIR, and its mechanism may be related to the suppression of post-SCIR autophagy.

SCIR results in the increased release of ROS, which induces OS and inflammatory responses and gives rise to neuronal apoptosis and autophagy, finally leading to irreversible neuronal injury if it is not treated in a timely manner. ${ }^{13}$ Research has verified that Apelin-13 has antiOS activity; in addition, it protects the ischemic myocardium, protects against cerebral ischemia reperfusion injury, and promotes smooth muscle cell proliferation. ${ }^{14}$ The results of this study demonstrated that the intraperitoneal injection of Apelin-13 pretreatment markedly improved the rat lower limb motor function, while the
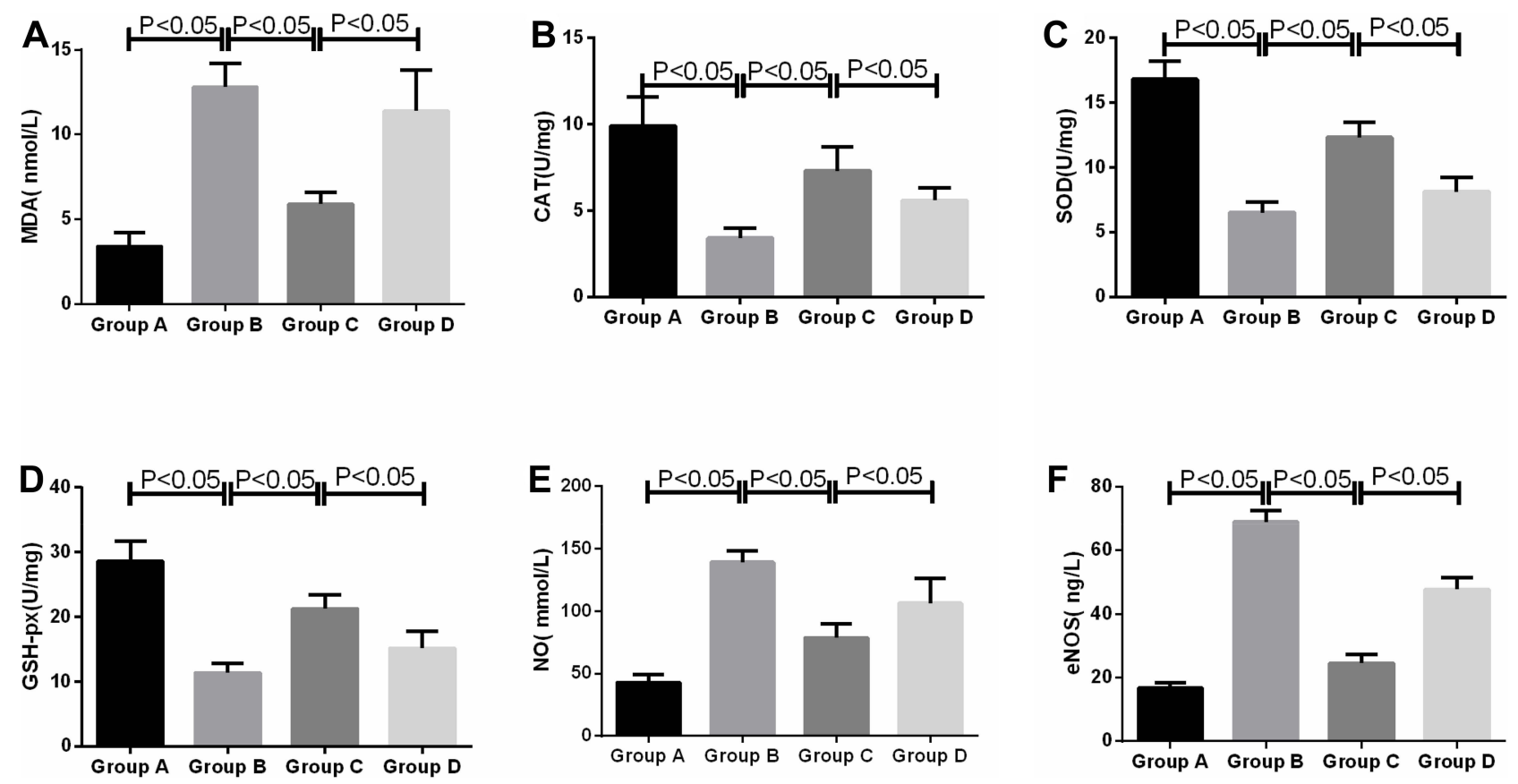

Figure 4 Comparison of OS factor expression levels in spinal cord tissues of four groups of rats $(n=8)$.

Notes: (A) MDA; (B) CAT; (C) SOD; (D) GSH-px; (E) NO; (F) eNOS. 

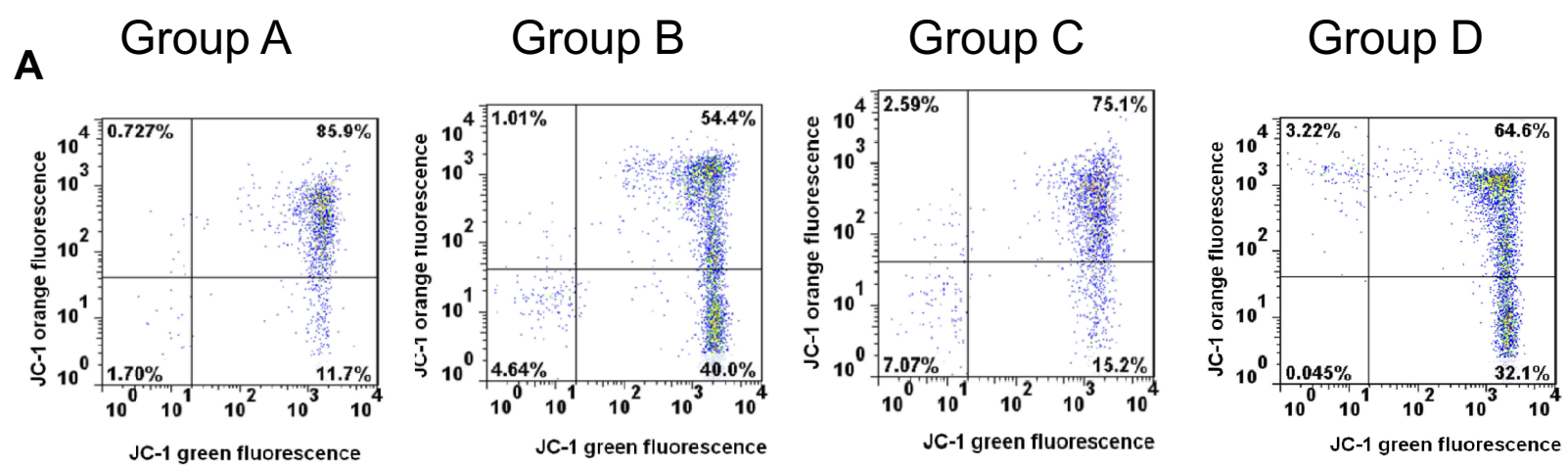

B
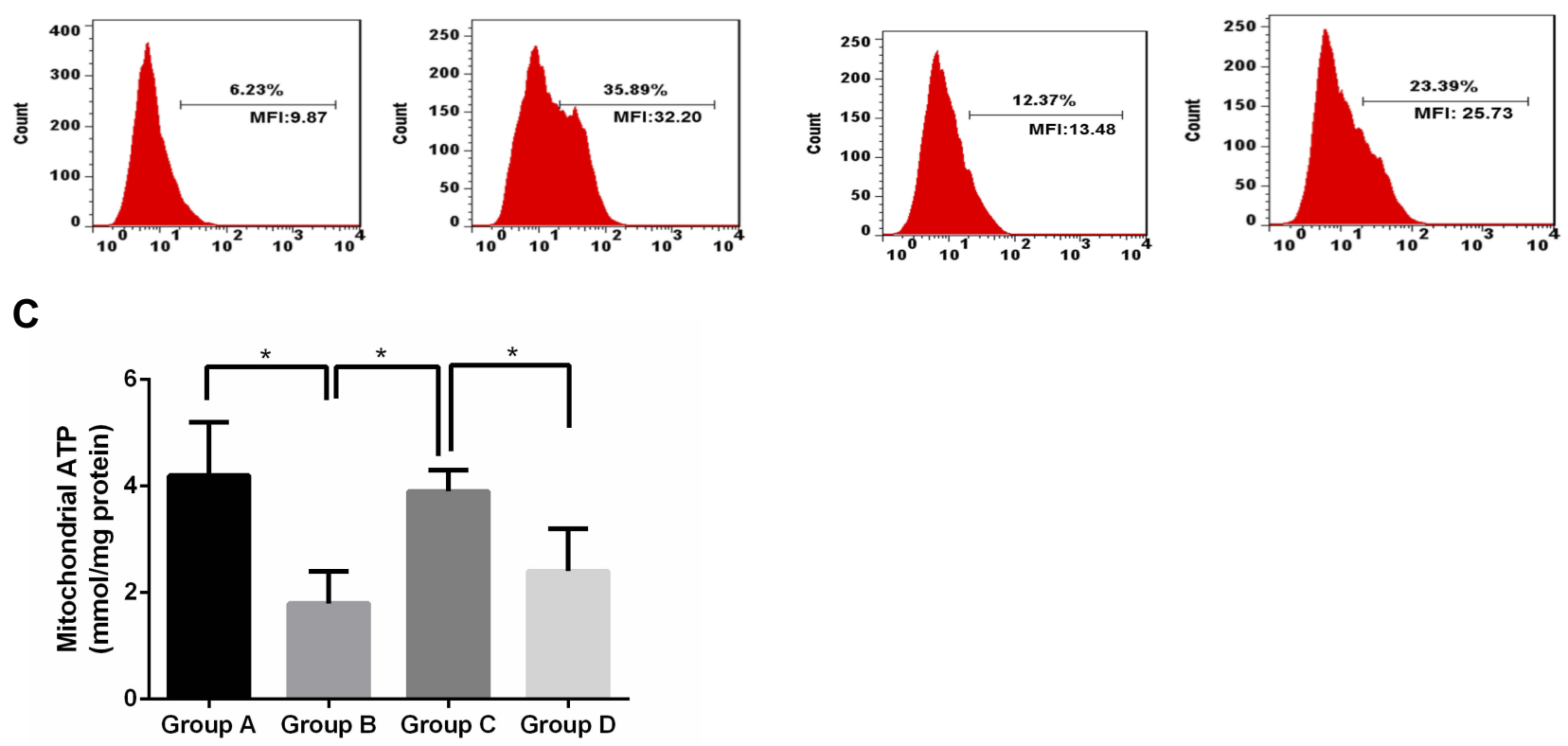

Figure 5 Effect of Apelin-13 pretreatment on ROS (A), MMP (B), and ATP content $(\mathbf{C})$ in SCIR-injured tissues $(n=8)$. Note: The asterisk $(*)$ indicates a significant difference $(P<0.05)$ between the two groups.
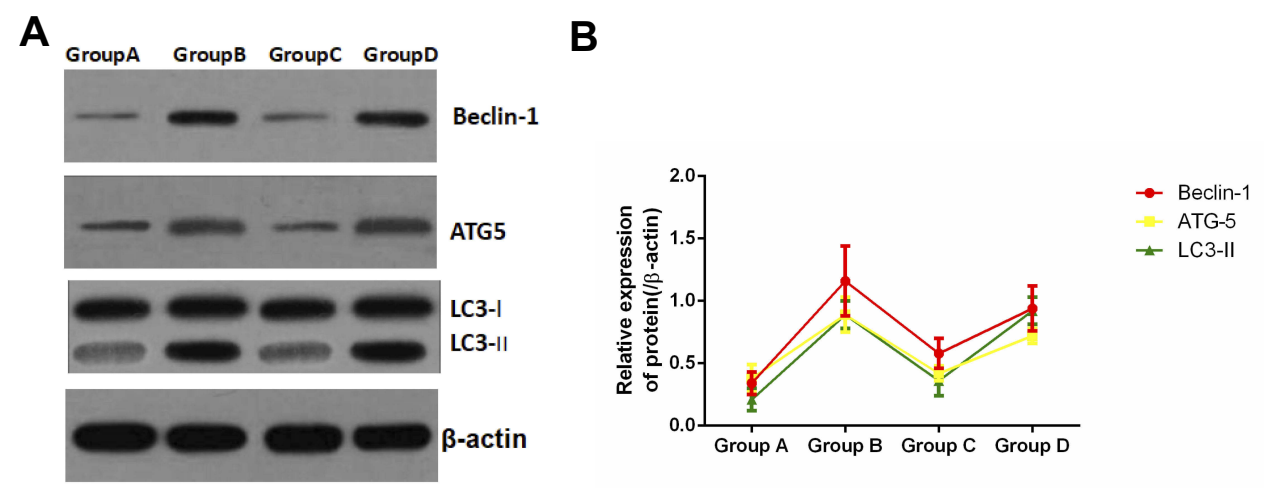

Figure 6 Effect of Apelin-13 pretreatment on the expression of autophagy-related proteins $(n=8)$.

Notes: (A) Typical electrophoretogram; (B) statistical analysis graph.

autophagy activator RAPA reversed the protective effect of Apelin-13, suggesting that the effect of Apelin-13 on SCIR may be related to autophagy.
After SCIR, the spinal cord tissues are in high OS status, which results in the release of tremendous amounts of ROS, while ROS destroy the polyunsaturated fatty acids in the cell 
membrane, produce lipid peroxide, and thereby injure tissues and cells. MDA is the metabolic end product of lipid peroxide, which reflects the ROS content and cell injury. ${ }^{15}$ CAT is the enzyme scavenger that replicates and removes ROS, thus avoiding ROS-induced cell injury. ${ }^{16}$ SOD is the primary enzyme of the antioxidase protection system in the body, which suppresses the production of lipid peroxide by lipid through a series of biochemical reactions, thereby preventing cell injury resulting from ROS. ${ }^{17} \mathrm{GSH}-$ $\mathrm{Px}$ is the catalyzing enzyme of $\mathrm{H}_{2} \mathrm{O}_{2}$ decomposition, and its activity in liver tissue is an important index that reveals the ROS scavenging capacity of the liver. ${ }^{18}$ This study detected the OS indices in four groups of rats on day 3 after SCIR. The results suggested that, compared with the sham operation group, the MDA content in the model group increased, while the activities of CAT, SOD, and GSH-Px were reduced, indicating that SCIR induced OS in spinal cord tissues. Meanwhile, the MDA content in the Apelin-13 pretreatment group was reduced, whereas the activities of CAT, SOD, and GSH-Px were enhanced, suggesting that Apelin-13 exerted antioxidative activity and reduced the OS status in spinal cord tissues. By contrast, RAPA and Apelin-13 pretreatment offset the antioxidation of Apelin-13, demonstrating that the activation of autophagy by the autophagy activator RAPA reversed the antioxidation of Apelin-13. NO and eNOS are extremely important physiological molecular signals, which participate in regulating numerous physiopathological processes, and their abnormal expression frequently induces vascular lesions. ${ }^{19}$ This study discovered that, on day 3 after SCIR, the levels of NO and eNOS in the model group were apparently higher than those in the sham operation group, while those in the Apelin-13 pretreatment group were markedly lower than those in the model group and evidently higher than those in the sham operation group, and those in the RAPA+Apelin-13 pretreatment group were remarkably higher than those in the Apelin-13 group. According to these findings, Apelin-13 showed anti-OS activity, which protected against SCIR-induced OS injury.

Apelin-13 is a physiologically bioactive peptide molecule that possesses favorable biocompatibility. Based on previous studies, Apelin-13 has multiple pharmacological activities, such as promoting vascular activity and protecting the nervous system, exerting a protective effect during the process of ischemia reperfusion injury in various organs. ${ }^{20,21}$ This study discovered, based on the SCIR model, that Apelin-13 pretreatment reduced the spinal cord water content, decreased the infarct volume, and lessened neuron death in model rats. These findings are similar to research results from other ischemia reperfusion organs, suggesting that Apelin-13 protected against the organ injury induced by ischemia reperfusion. $^{22,23}$

The mitochondrion is the site for ATP synthesis, while ATP provides the energy source for cellular activities, and is synthesized through the mitochondrial respiratory chainmediated redox reaction. Consequently, numerous diseases, including SCIR, are related to abnormal ATP synthesis resulting from mitochondrial dysfunction. ${ }^{24}$ As discovered in previous studies, dysfunction of the mitochondrial respiratory chain complex at the time of ischemia usually results in impaired MMP, which leads to increased release of ROS and aggravates cell damage. ${ }^{25}$ Our results suggested that, compared with the sham operation group, MMP in spinal cord tissues of the model group was destroyed, ROS production increased, and ATP synthesis was reduced, while mitochondrial function destruction in the Apelin-13 pretreatment group was markedly alleviated, revealing that the effect of Apelin-13 in protecting spinal cord tissues from SCIR was related to the protection of mitochondrial function.

The autophagy phenomenon is considered as an intracellular catabolic and degradation mechanism for abnormal cell contents in cells under energy crisis through the autophagosome-lysosome pathway. This mechanism contributes toward maintaining cellular homeostasis, and is related to cell autophagic death, also known as programmed cell death. Existing studies have proved that, in the case of organ ischemia reperfusion, the autophagy phenomenon abnormally increases. Takaesu et al suggested that autophagy at a reasonable level scavenges the pathological organelles in cells, and facilitates the maintenance of cell survival, but abnormally increased autophagy may result in cell death. ${ }^{26}$ The role of autophagy in the ischemia reperfusion process has attracted increasing attention from scholars. Gurusamy et al, in their research on myocardial ischemia reperfusion injury, indicated that, after ischemia and reperfusion, the expression of autophagy-related proteins LC3-II and ATG5 was remarkably up-regulated. ${ }^{27}$ This study also adopted the Western blotting assay to detect the expression of autophagy-related proteins Beclin1, Atg5, and LC3 in spinal cord tissues of four groups of rats. The results revealed that the expression of these three autophagyrelated proteins in spinal cord tissues of the model group was evidently up-regulated, while it was markedly lower in the Apelin-13 pretreatment group than in the model group, and that in the RAPA+Apelin-13 pretreatment group was lower than that in the Apelin-13 pretreatment group. Such research results further verified that, at the time of ischemia 
reperfusion injury, the autophagy level in spinal cord tissues was abnormally elevated, while Apelin-13 suppressed autophagy to protect against SCIR, and the autophagy activator antagonized the protective activity of Apelin-13.

\section{Conclusion}

To sum up, this study constructs an SCIR model and discovers that the autophagy level in spinal cord tissues abnormally increases after SCIR, while Apelin-13 pretreatment suppresses the expression of the autophagy-related proteins LC3-II, Beclin1, and ATG5. These results indicate that Apelin-13 exerts a protective effect on SCIR by suppressing autophagy, while the addition of an autophagy activator antagonizes the protective effect of Apelin-13, further proving that Apelin-13 offers protection from SCIR by inhibiting autophagy. In conclusion, the animal experiment presented in this study verifies that Apelin-13 pretreatment confers protection from SCIR, and its mechanism of action is related to the suppression of autophagy. This study has provided new ways of thinking and new clues for developing therapeutics to reduce perioperative SCIR.

\section{Disclosure}

The authors report no conflicts of interest in this work.

\section{References}

1. Jiang X, Ai C, Shi E, Nakajima Y, Ma H. Neuroprotection against spinal cord ischemia-reperfusion injury induced by different ischemic postconditioning methods: roles of phosphatidylinositol 3-kinase-Akt and extracellular signal-regulated kinase. Anesthesiology. 2009;111 (6):1197-1205. doi:10.1097/ALN.0b013e3181bf1d93

2. Elmore JR, Gloviczki P, Harper CM Jr, et al. Spinal cord injury in experimental thoracic aortic occlusion: investigation of combined methods of protection. J Vasc Surg. 1992;15(5):789-798.

3. Francel PC, Long BA, Malik JM, Tribble C, Jane JA, Kron IL. Limiting ischemic spinal cord injury using a free radical scavenger 21-aminosteroid and/or cerebrospinal fluid drainage. J Neurosurg. 1993;79(5):742-751. doi:10.3171/jns.1993.79.5.0742

4. Parrino PE, Kron IL, Ross SD, et al. Retrograde venous perfusion with hypothermic saline and adenosine for protection of the ischemic spinal cord. J Vasc Surg. 2000;32(1):171-178. doi:10.1067/ mva.2000.105003

5. Wang JL, Xu CJ. Astrocytes autophagy in aging and neurodegenerative disorders. Biomed Pharmacother. 2019;122:109691. doi:10.1016/ j.biopha.2019.109691

6. Roca-Agujetas V, de Dios C, Lestón L, Marí M, Morales A, Colell A. Recent insights into the mitochondrial role in autophagy and its regulation by oxidative stress. Oxid Med Cell Longev. 2019;2019:3809308. doi:10.1155/2019/3809308

7. Tang P, Hou H, Zhang L, et al. Autophagy reduces neuronal damage and promotes locomotor recovery via inhibition of apoptosis after spinal cord injury in rats. Mol Neurobiol. 2014;49(1):276-287.

8. Zhang HY, Wang Z, Wu F, Kong X. Regulation of autophagy and ubiquitinated protein accumulation by bFGF promotes functional recovery and neural protection in a rat model of spinal cord injury. Mol Neurobiol. 2013;48(3):452-464. doi:10.1007/s12035-013-8432-8
9. Yamaleyeva LM, Shaltout HA, Varagic J. Apelin-13 in blood pressure regulation and cardiovascular disease. Curr Opin Nephrol Hypertens. 2016;25(5):396-403. doi:10.1097/MNH.0000000000000241

10. Bao H, Han W, Zhang L, Dai D. Apelin-13 attenuates traumatic brain injury-induced damage by suppressing autophagy. Neurochem Res. 2015;40(1):89-97. doi:10.1007/s11064-014-1469-x

11. Liu S, Qu Y, Stewart TJ, et al. Embryonic stem cells differentiate into oligodendrocytes and myelinate in culture and after spinal cord transplantation. PNAS. 2000;97(11):6126-6131. doi:10.1073/ pnas.97.11.6126

12. Basso DM, Beattie MS, Bresnahan JC . Graded Histological and Locomotor Outcomes after Spinal Cord Contusion Using the NYU Weight-Drop Device versus Transection. Exp Neurol. 1996;139(2):0256. doi10.1006/exnr.1996.0098

13. Song W, Sun J, Su B, Yang R, Dong H, Xiong L. Ischemic postconditioning protects the spinal cord from ischemia-reperfusion injury via modulation of redox signaling. $J$ Thoracic Cardiovasc Surg. 2013;146(3):688-695. doi:10.1016/j.jtcvs.2012.11.039

14. Simpkin JC, Yellon DM, Davidson SM, Lim SY, Wynne AM, Smith CCT. Apelin-13 and apelin-36 exhibit direct cardioprotective activity against ischemia-reperfusion injury. Basic Res Cardiol. 2007;102(6):518-528. doi:10.1007/s00395-007-0671-2

15. Gavella M, Lipovac V, Rako R, olak B. Reduction of oxidative changes in human spermatozoa by exogenous gangliosides. Andrologia. 2005;37 (1):17-24. doi:10.1111/j.1439-0272.2004.00646.x.

16. Hu P, Tirelli N. Scavenging ROS: superoxide dismutase/catalase mimetics by the use of an oxidation-sensitive nanocarrier/enzyme conjugate. Bioconjug Chem. 2012;23(3):438-449. doi:10.1021/ bc200449k

17. Younus H. Therapeutic potentials of superoxide dismutase. Int J Health Sci. 2018;12(3):88-93.

18. Margis R, Dunand C, Teixeira FK, Margis-Pinheiro M. Glutathione peroxidase family - an evolutionary overview. FEBS J. 2008;275 (15):3959-3970. doi:10.1111/j.1742-4658.2008.06542.x

19. Zhao Y, Vanhoutte PM, Leung SW. Vascular nitric oxide: beyond eNOS. J Pharmacol Sci. 2015;129(2):83-94. doi:10.1016/j. jphs.2015.09.002

20. Tao J, Zhu W, Li Y, et al. Apelin-13 protects the heart against ischemia-reperfusion injury through inhibition of ER-dependent apoptotic pathways in a time-dependent fashion. Am $J$ Physiol. 2011;301(4):H1471. doi:10.1152/ajpregu.00756.2010

21. Li L, Zeng H, Chen JX. Apelin-13 increases myocardial progenitor cells and improves repair postmyocardial infarction. Am J Physiology Heart Circulatory Physiol. 2012;303(5):H605-18. doi:10.1152/ ajpheart.00366.2012

22. Rastaldo R, Cappello S, Folino A, Berta GN, Pagliaro P. Apelin-13 limits infarct size and improves cardiac postischemic mechanical recovery only if given after ischemia. AJP Heart Circulatory Physiol. 2011;300(6):H2308-15. doi:10.1152/ajpheart.01177.2010

23. Liu XY, Lu Q, Ouyang X, et al. Apelin-13 increases expression of ATP-binding cassette transporter A1 via activating protein kinase $\mathrm{C} \alpha$ signaling in THP-1 macrophage-derived foam cells. Atherosclerosis. 2013;226(2):398-407. doi:10.1016/j.atherosclerosis.2012.12.002

24. Bao L, Abe K, Tsang P, et al. Bilberry extract protect restraint stress-induced liver damage through attenuating mitochondrial dysfunction. Fitoterapia. 2010;81(8):1094-1101. doi:10.1016/j. fitote.2010.07.004

25. Cardoso SM, Pereira C, Oliveira CR. Mitochondrial function is differentially affected upon oxidative stress. Free Radic Biol Med. 1999;26(1-2):3-13. doi:10.1016/S0891-5849(98)00205-6

26. Takaesu G, Kobayashi T, Yoshimura A. TGF $\beta$-activated kinase 1 (TAK1)-binding proteins (TAB) 2 and 3 negatively regulate autophagy. J Biochem. 2012;151(2):157-66. doi: 10.1093/jb/mvr123.

27. Gurusamy N, Das DK. Autophagy, redox signaling, and ventricular remodeling. Antioxid Redox Signal. 2009;11(8):1975-1988. doi:10.1089/ars.2009.2524 


\section{Publish your work in this journal}

Drug Design, Development and Therapy is an international, peerreviewed open-access journal that spans the spectrum of drug design and development through to clinical applications. Clinical outcomes, patient safety, and programs for the development and effective, safe, and sustained use of medicines are a feature of the journal, which has also been accepted for indexing on PubMed Central. The manuscript management system is completely online and includes a very quick and fair peer-review system, which is all easy to use. Visit http://www. dovepress.com/testimonials.php to read real quotes from published authors.

Submit your manuscript here: https://www.dovepress.com/drug-design-development-and-therapy-journal 